\title{
FEMTOSECOND TRANSIENT ABSORPTION MICROSPECTROSCOPY OF BENZIL CONFINED INTO A SINGLE BEAD OF POROUS GLASS
}

\author{
CHRISTOPHER F. PORTER ${ }^{1}$, NAOTO TAMAI ${ }^{2}$ \\ and HIROSHI MASUHARA ${ }^{3}$ \\ Microphotoconversion Project ${ }^{4}$, ERATO, Research Development \\ Corporation of Japan, 15 Morimoto-cho, Shimogamo, \\ Sakyo-ku, Kyoto 606, Japan \\ (Received 27 September, 1995)
}

\begin{abstract}
The photophysical properties of benzil dissolved in free solution and in solution confined into a single bead of porous glasses (size $<100 \mu \mathrm{m}$ ) were investigated by femtosecond transient absorption microspectroscopy. In nonpolar solvent, cyclohexane, the rate of conformational change from the skewed to the near trans-planar form was reduced from $2.2 \times 10^{12} \mathrm{~s}^{-1}$ in free solution to $9.1 \times 10^{11} \mathrm{~s}^{-1}$ in porous glasses with an average pore diameter of $26 \mathrm{~nm}$. In polar solvent, DMF, however, there was little difference between the two systems.
\end{abstract}

KEY WORDS: Benzil, Porous glass, Femtosecond, Transient absorption, Microspectroscopy.

\section{INTRODUCTION}

Considerable attention has been paid to the investigations of the structure and dynamics in porous glass systems. One interesting subject for the structure is the fractal nature of porous glasses, ${ }^{1-5}$ which is investigated by, amongst other methods, Förster-type excitation energy transfer experimenets between adsorbed donors and acceptors. The surfaces of porous glass walls themselves have also been interrogated by many other groups. ${ }^{6-8}$ Fujii et al. studied the photophysical properties of pyrene molecules adsorbed on porous glasses by the technique of time-resolved fluorescence spectroscopy and proposed that the significant interactions with hydroxyl groups of different polarity were responsible for the observed complexity of pyrene photophysics. ${ }^{7}$ To give consideration to the nature of the environments existing in the porous glass, Hench and West suggested that at room temperature,

\footnotetext{
${ }^{1}$ Present Address: School of Environmental Sciences, University of East Anglia, Norwich NR4 7TJ, England.

${ }^{2}$ Present Address: Light and Material Group, PRESTO, JRDC; Department of Chemistry, Kwansei Gakuin University, Uegahara, Nishinomiya 662, Japan.

${ }^{3}$ Permanent Address: Department of Applied Physics, Osaka University, Osaka 565, Japan.

${ }^{4}$ Five-year term project (Oct. 1988-Sept. 1993). All correspondence should be sent to H. M. at Osaka University.
} 
water is hydrogen bonded to silanol groups. ${ }^{8}$ On increasing temperature to $170^{\circ} \mathrm{C}$, the adsorbed water is removed from the porous glass and adjacent silanol groups bridge with each other through their hydrogen atoms.

Another subject of interest is dynamic behavior of liquids confined in the porous glass, which is related to fluidity, translational and rotational diffusion, and melting behavior of liquids. ${ }^{9-13}$ Neutron scattering experiments of water confined in porous glass revealed the presence of a small amount of liquid water even at temperatures down to $-40^{\circ} \mathrm{C} .{ }^{13}$ The reduction of self-diffusion by 2 orders of magnitude for azobenzene was also demonstrated in the porous glass system filled with alcoholtoluene mixtures. ${ }^{10}$

The present paper seeks to address the environmental effects of porous glasses on benzil solutions, confined in small domains, by femtosecond transient absorption microspectroscopy. It is reported that the geometry of benzil in the ground state is skewed so as to take a dihedral angle between the planes containing the benzoyl groups to be $98^{\circ}$ (in benzene at room temerature). ${ }^{14}$ This probe molecule, on excitation, undergoes conformational change from the skewed to the near transplanar form, the rate of which is dependent on solvent viscosity and geometric restrictions. The advantage of the use of this molecule is the spectral simplicity and clarity of the intramolecular structural change. Its disadvantage is that at room temperature, this process occurs on a picosecond $\sim$ subpicosecond time scale, ${ }^{15}$ thus making conventional fluorescence detection techniques impractial to use. By the femtosecond transient absorption spectroscopy, conformational dynamics can be directly followed and analyzed.

Since porous glasses are usually available as powder with a diameter less than a hundred $\mu \mathrm{m}$, conventional transmittance transient absorption spectroscopy cannot be adapted to such a system. For optically scatting systems consisting of an assembly of glass beads, time-resolved diffuse reflectance spectroscopy has been widely applied to analyze the photophysical and photochemical dynamics in confined systems. ${ }^{16-18}$ It was reported that, however, the temporal resolution of a time-resolved diffuse reflectance system was dramatically reduced from femtosecond to a few picosecond time scales depending on the scattering and absorption coefficient in the case that the femtosecond laser pulse was utilized. ${ }^{19}$ This method, therefore, is not easily adapted to the current experimental systems. In addition, measurements of a single porous glass bead will be better owing to its narrow diameter dispersion than an assembly of glass beads.

In the present work, a newly developed transient absorption spectrometer combined with a microscope was applied to the porous glass/benzil solution system, and femtosecond $\sim$ picosecond dynamics in a single micrometer-sized glass bead was investigated for the first time. Rates of conformatinoal change of benzil confined into a single porous glass bead were analyzed in comparision with those in the bulk solution.

\section{EXPERIMENTAL}

Benzil (Tokyo Kasei, zone refined), cyclohexane and N, N-dimethylformamide (DMF) (Nacalai Tesque, spectroscopic grade) were used as received. The porous 
glass (Fuji-Davidson Chemical Co.) of $26 \mathrm{~nm}$ pore size with narrow diameter distribution $(< \pm 7 \%$, measured by Mercury porosimetry at the company) was heated to around $380 \mathrm{~K}$ under vacuum for approximately three hours, then benzil solution was promptly added to the cell containing porous glasses. The diameter of a porous glass bead was less than $100 \mu \mathrm{m}$. Since no modifications to the porous glass surfaces were carried out, a significant density of silanol groups are expected to have been present.

Femtosecond transient absorption spectroscopy with micrometer spatial resolution ${ }^{20}$ was utilized and a brief outline of the experimental set up is as follows. The excitation beam $(\sim 360 \mathrm{~nm})$ was generated by frequency doubling the amplified output of the dye laser (Coherent, Satori 774). The gain dye used in the synchronously pumped, hybridly mode-locked dye laser was pyridine 2. Pyridine 1 was employed as the gain dye used for the three stage regenerative amplifier (Continuum, RGA 60) system. Insertion of a saturable absorber filter between the first and second stage of the amplifier resulted in the pulse width being of $150 \sim 200$ fs FWHM without side wings. The net output was a train of pulses with a repetition rate of $10 \mathrm{~Hz}$, a center wavelength of $720 \mathrm{~nm}$, and a pulse energy of approximately $400 \mu \mathrm{J} /$ pulse. For the generation of the white light continuum to be used as an optical probe, the residual amplified fundamental dye laser beam was focused into a 1-cm water filled cuvette.

To measure the transient absorption spectra of the free solutions (i.e. not confined into porous glasses), the sample was placed in a $2-\mathrm{mm}$ cuvette and a conventional optical arrangement were utilized. For the investigation of the porous glass systems, a modified microscope was used in conjunction with the laser system, thus facilitating the interrogation of a single porous glass bead of approximately $20 \sim 50 \mu \mathrm{m}$ diameter. For these experiments, a 15-X reflective objective lens was used, which is characteristically less susceptible to chromatic aberration. Furthermore, the time dispersion of the white-light continuum in femtosecond transient absorption spectroscopy is smaller than that of the conventional microscope.

The white-light continuum was split into probe and reference beams, each of which was imaged onto separate quartz fibers coupled to the input slits of the two polychromators (McPherson 2035) and detected with multichannel photodiode arrays (Princeton Instruments PDA-1024 and ST-1000). The spectra were corrected for time dispersion of the probe pulse thereby facilitating temporal resolution of the early gated spectra. All measurements were carried out at $293 \pm 2 \mathrm{~K}$.

\section{RESULTS AND DISCUSSION}

Figure 1a illustrates transient absorption spectra of benzil in bulk cyclohexane solution at various probe delays. The spectrum just after excitation $(-0.2 \sim-0.1$ ps) shows a broad spectrum with a peak at approximately $570 \mathrm{~nm}$. With increasing delay time, the spectrum shifted rapidly to the blue wavelength region, and its width became narrower with time. The transient absorption spectrum at long delay time region had a peak at approximately $530 \mathrm{~nm}$. For analyzing the spectral shift more precisely, a contour plot of transient absorption spectra was illustrated in Figure $1 \mathrm{~b}$. 

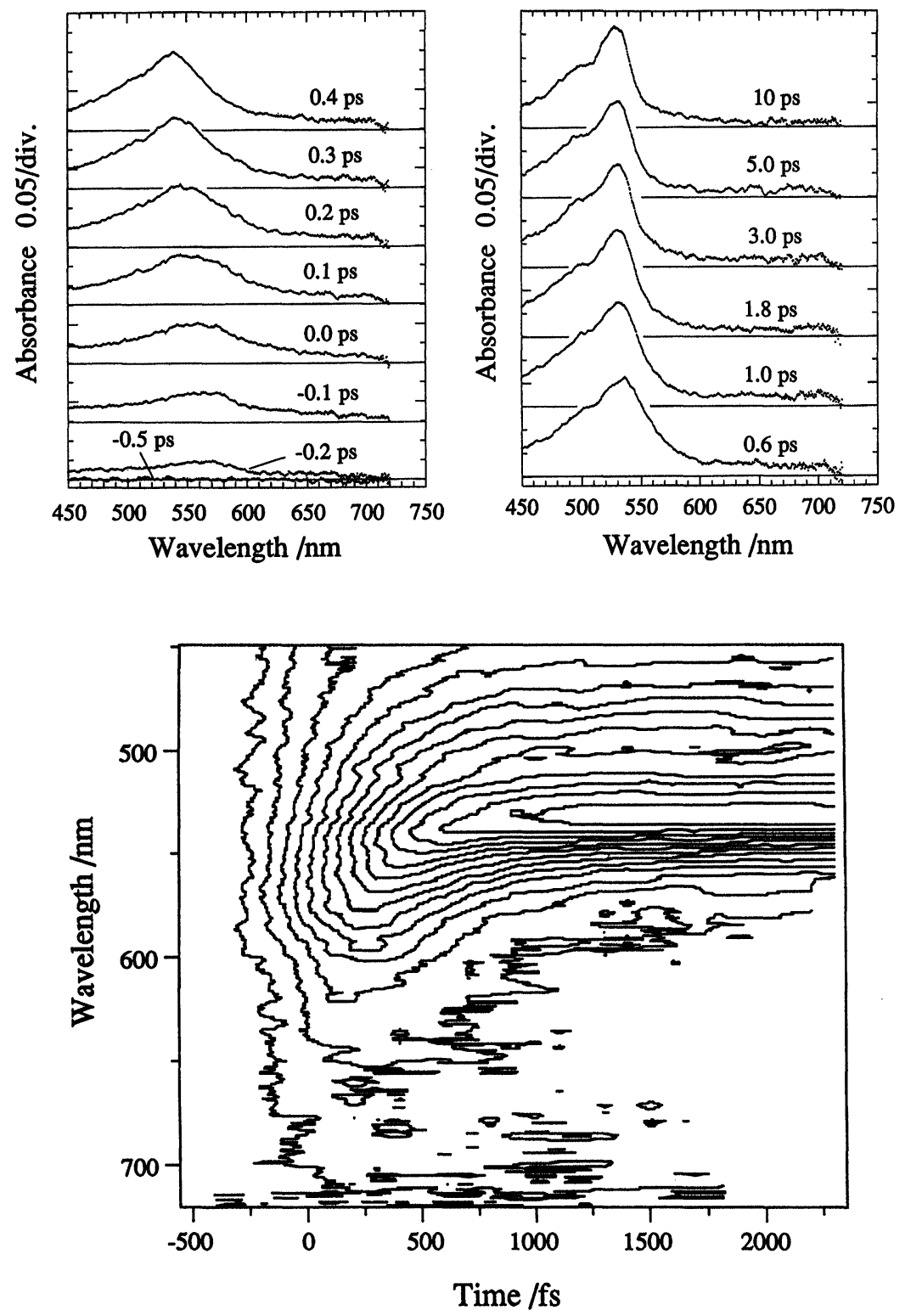

Figure 1 (a) Transient absorption spectra of benzil dissolved in cyclohexane. Delay times are written in the Figure. (b) Contour plot of transient absorption spectra for Figure 1.

As clearly shown, the spectral shift finished within 1.0 ps after excitation, in which the absorption maximum was located at $\sim 530 \mathrm{~nm}$. The spectrum with a delay time longer than $1 \mathrm{ps}$ is safely assigned to the $\mathrm{S}_{\mathrm{n}} \leftarrow \mathrm{S}_{1}$ absorption of the near trans-planar form of benzil. ${ }^{21}$ The spectrum just after the excitation with a peak at $\sim 570 \mathrm{~nm}$ 
probably corresponds to the $S_{n} \leftarrow S_{1}$ absorption from the unrelaxed skewed form of benzil. This is supported from the fact that the current spectral shape and its peak wavelength are similar to the $S_{n} \leftarrow S_{1}$ absorption of benzil crystal being assigned to that of skewed form obtained by a picosecond diffuse reflectance laser photolysis method. ${ }^{21}$

To analyze the rate of conformational change, the peak energy of transient absorbance, and the absorbance at $480 \mathrm{~nm}$ and $580 \mathrm{~nm}$ were plotted as a function of delay time as shown in Figure 2. The decay time constant observed at $580 \mathrm{~nm}$ was estimated to be $\sim 440 \mathrm{fs}$, which was in agreement with the rise time constant observed at $480 \mathrm{~nm}(\sim 500 \mathrm{fs})$ within experimental error. Furthermore, the rise and decay time constants were very close to the value for the spectral shift as shown in Figure $2 \mathrm{a}$, which was $\sim 430 \mathrm{fs}$ by assuming a single exponential function. From these results, the rate of the conformational change of benzil in free cyclohexane solution was estimated to be approximately $2.2 \times 10^{12} \mathrm{~s}^{-1}$.

This experiment in solution is essentially similar to the work reported by Miyasaka and Mataga ${ }^{15}$ and will be used as a reference for the work on porous glass systems. They found that the transient absorption spectrum of benzil just after the excitation had a peak at $545 \sim 550 \mathrm{~nm}$ in $\mathrm{n}$-hexane and cis-decalin solution, and the rate of conformational change of the excited benzil was $\sim 2 \mathrm{ps}$ in cyclohexane.
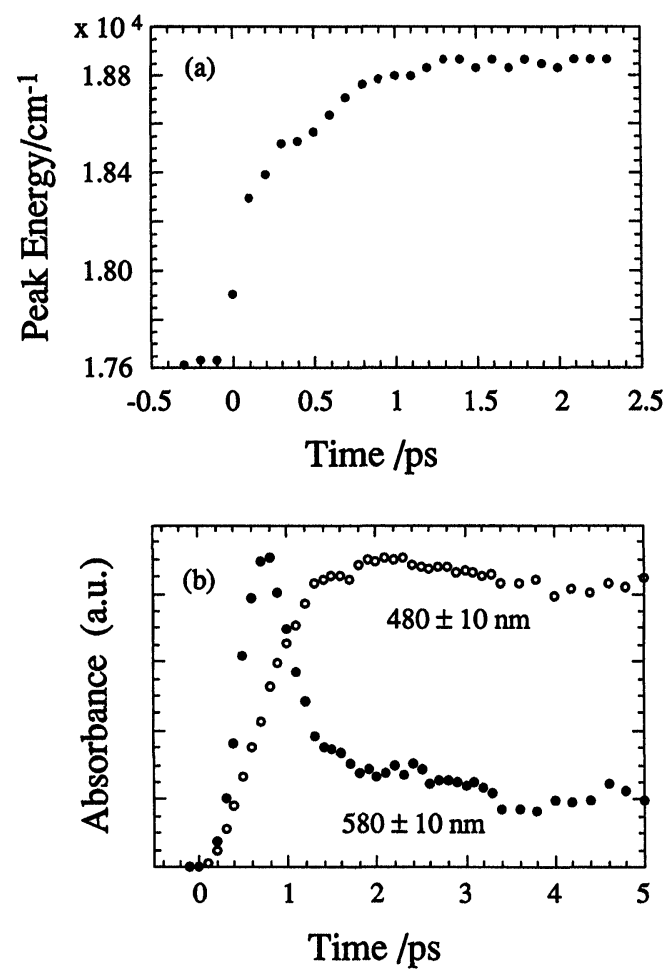

Figure 2 (a) Peak wavelength of transient absorption spectra as a function of delay time. (b) Rise and decay curves of absorbance recorded at $480 \pm 10 \mathrm{~nm}$ and $580 \pm 10 \mathrm{~nm}$. 
They also found the linear relationship between the time constant of conformational change and the solvent viscosity. Our result on the conformational change in cyclohexane solution, however, is faster than that obtained by Miyasaka and Mataga. A possible reason for the discrepancy is that their probe wavelength was set to 550 $\mathrm{nm}$ with the result that their transient absorption decay curves have significantly larger contribution of the near trans-planar absorption, which may have led to increased uncertainty. An another possible reason may be due to the difference in the excitation pulse width, but the reason still remains unclear.

Figure 3 shows the transient absorption spectra of a single porous glass bead saturated with concentrated benzil/cyclohexane solution. Although the $\mathrm{S} / \mathrm{N}$ ratio of the spectra is relatively low because of the small thickness of the porous glass, the following results were obtained. The transient absorption spectrum just after excitation can be interpreted as the skewed form, because its peak wavelength is located at $\sim 570 \mathrm{~nm}$. The spectrum shifted to the blue wavelength region with time being similar to the free solution. Even at $0.80 \mathrm{ps}$, the spectral profile of the skewed form is still detected, however, when the probe was set to a delay of $2.4 \mathrm{ps}$, the resulting transient absorption spectrum is dominated by the near trans-planar form. Finally, the spectrum at $\sim 12$ ps delay time is similar to the late spectrum of free solution. This suggests that the effects of confinement in the porous glass system still allows conformational change, but at a reduced rate compared to the bulk solution. In addition, it is considered that benzil molecules are not actually adsorbed (or chemisorbed) onto the porous glass walls, because the spectral shapes of transient absorption corresponding to the singlet state of the skewed and near trans-planar forms for both the free solutions and porous glass systems are very similar. Figure 4 illustrates transient absorption decay curve of benzil/cyclohexane solution confined
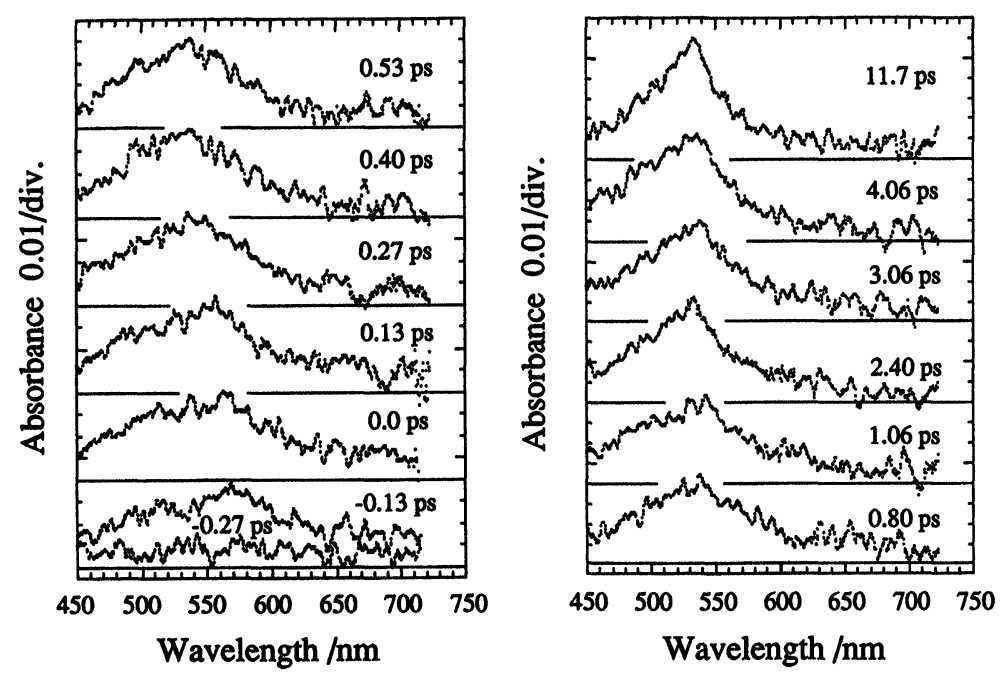

Figure 3 Transient absorption spectra of a single porous glass bead of $26 \mathrm{~nm}$ pore size saturated with benzil/cyclohexane solution. 


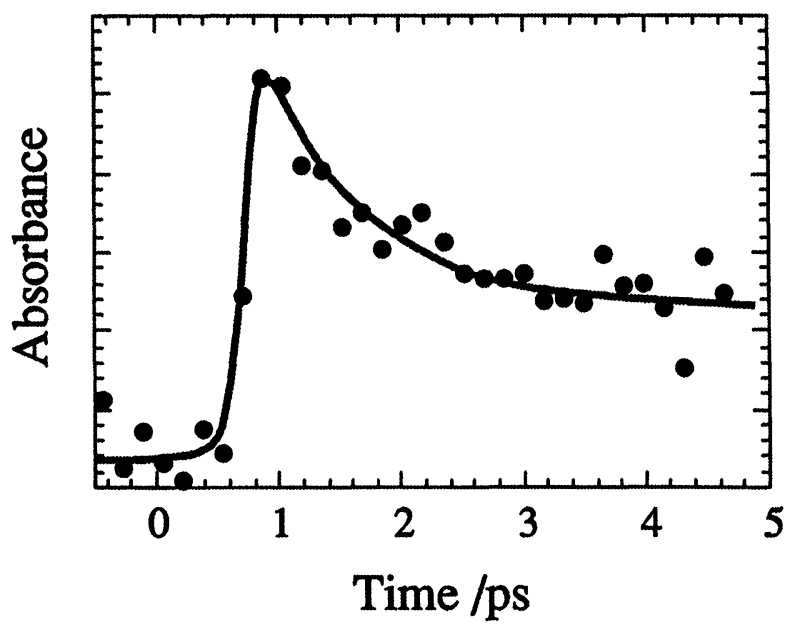

Figure 4 Decay curve of transient absorption of benzil/cyclohexane solution confined into porous glass recorded at $580 \pm 10 \mathrm{~nm}$ region.

into porous glass recorded at $580 \pm 10 \mathrm{~nm}$ region. The decay time constant in the porous glass system was estimated to be $\sim 1.1 \mathrm{ps}$, which is approximately two times slower than that in free solution. From these results, the rate of conformational change of benzil in nonpolar cyclohexane solvent confined into a porous glass was found to be $\sim 9.1 \times 10^{11} \mathrm{~s}^{-1}$ which is slower than the bulk solution.

For further understanding the dynamics in confined system, benzil kinetics were investigated using DMF which is polar and a thermodynamically good solvent. Figures 5 and 6 illustrate the transient absorption spectra of free DMF solution and the porous glass system, respectively. In both systems, the spectrum just after excitation has a peak at $\sim 580 \mathrm{~nm}$ and its maximum shifts gradually to the blue wavelength region. Finally, the spectrum has a peak at $\sim 530 \mathrm{~nm}$ corresponding to the near trans-planar form of benzil in the excited singlet state. The temporal behavior of both systems was similar to each other. To analyze the rate of conformational change, transient absorption decay curves recorded at $580 \pm 10 \mathrm{~nm}$ region were plotted in Figure 7. The decay time constants in both free solution and porous glasses were found to be identical to each other and estimated to be $700 \sim 800$ fs (the rate of conformational change $\sim 1.3 \times 10^{12} \mathrm{~s}^{-1}$ ).

One explanation for the present result is that since benzil is saturated in cyclohexane with rather low solubility, in solution being confined into the porous glasses, the benzil molecules may be concentrated in the vicinity of the polar glass walls. The effective pore size may then be perceived to the sufficiently reduced to the extent where the rate of conformational change becomes affected. This is compatible with the report by Dierker et al. in their investigations of a binary liquid mixture adsorbed into porous glass. ${ }^{22}$ For these investigations, they used a Raman scattering technique to study wetting and fluid flow and realized the preferential concentration of one of the components in the vycor glass. 

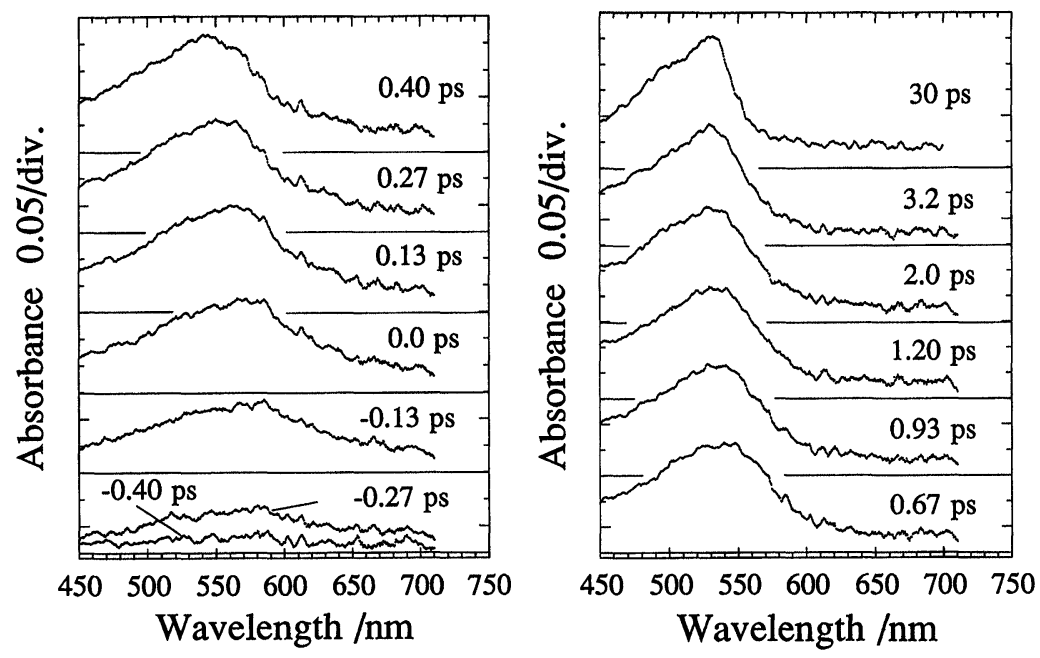

Figure 5 Transient absorption spectra of benzil dissolved in DMF. Delay times are written in the Figure.
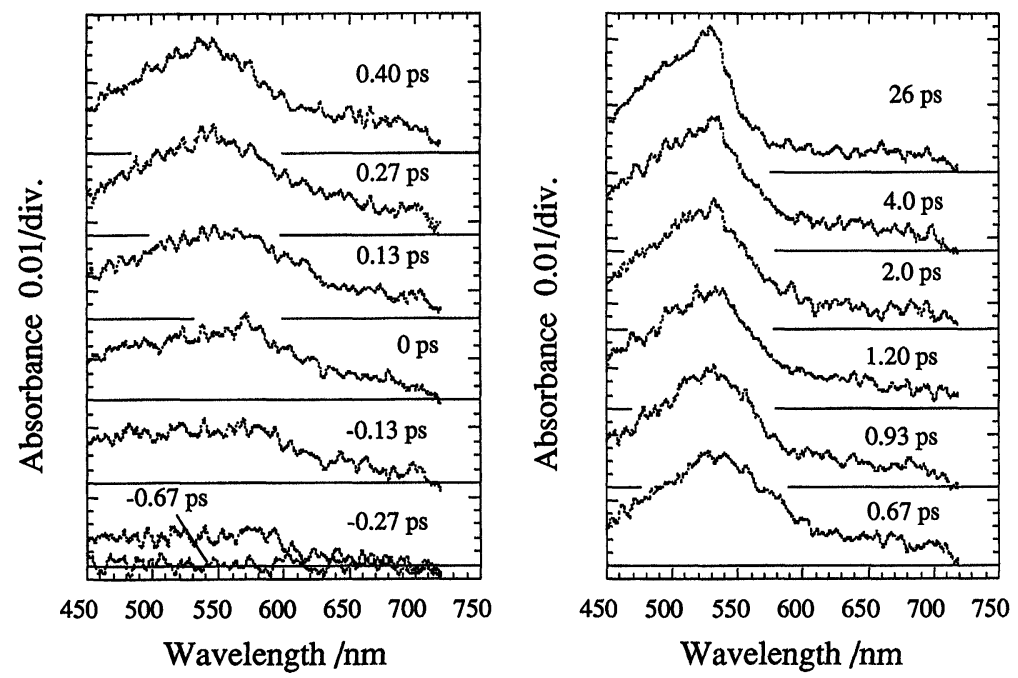

Figure 6 Transient absorption spectra of benzil/DMF solution confined into a single porous glass bead of $26 \mathrm{~nm}$ pore size. Delay times are written in the Figure.

On the other hand, DMF has a high solubility for benzil, so that such an effect may not be observed. This is consistent with the results concerning a DMF system given by Drake and Klafter. ${ }^{1}$ They reported that the rotational anisotropy of rhodamine $6 \mathrm{G}$ in solution, confined into porous glass, was significantly reduced when a pore size of $4 \mathrm{~nm}$ or below was utilized. We used here the porous glass with $26 \mathrm{~nm}$ 

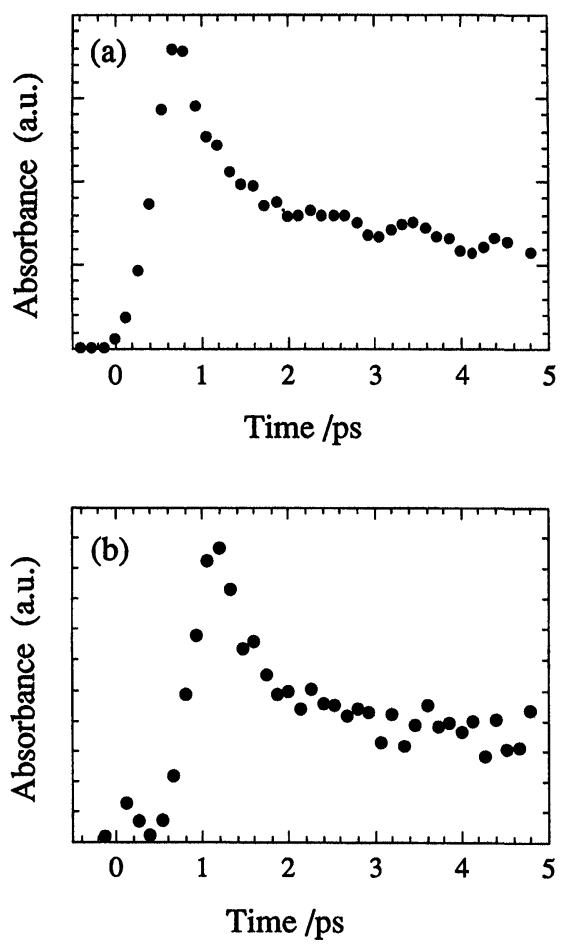

Figure 7 Decay curve of transient absorption of benzil/DMF in free solution (a) and in a sigle porous glass (b) recorded at $580 \pm 10 \mathrm{~nm}$ region.

pore size. In addition, it would be unreasonable to assume that benzil has a lower hydrodynamic volume compared to rhodamine $6 \mathrm{G}$.

Another possible explanation considers the surface of the porous glass walls. Since the porous glass was not treated, a significant population density of silanol groups are likely to be present. In addition, since the porous glass was heated to only around $380 \mathrm{~K}$, adsorbed water is expected to be present to at least a monolayer depth. The purpose of the heat treatment was simply to remove bulk water from the glass pores in order to facilitate penetration of the sample solutions into pores. The wall of porous glass is probably highly polar and significantly affects the photophysical properties of benzil. As cyclohexane is a nonpolar solvent, benzil with two carbonyl groups may preferentially interact with the surface of porous glasses by hydrogen bonding. This effect presumably leads to the corresponding reduction in the rate constant of conformational change as compared with the bulk solution. The strong interaction of benzil on the glass wall is probably excluded as discussed in the following. It is reported that the adsorption of malachite green at the quartz/aqueous solution interface induces a significant increase in the excited singlet-state lifetime by more than two-orders of magnitude, indicating that the rate of torsional motion of the phenyl ring of malachite green is reduced by over two-orders of 
magnitude, by the adsorption, as compared with that in free solution. ${ }^{23}$ In the current experiments on porous glass systems, the transient absorption spectrum in the late time region is very similar to that in free solution, and furthermore, the rate of conformational change in the confined system is only one half of the free solution. This result suggests that the strong interaction of benzil on the glass wall is negligible. The effect of hydrogen bonding on polar glass surfaces is less probable in polar DMF. Actually, the current result in DMF solution suggest that the effect of the polarity of the porous glass walls on molecular reorientation is undetectably small.

In conclusion, femtosecond transient absorption spectra of molecules in porous glass systems were observed for the first time by measuring a single glass bead using a microscope. In the case of cyclohexane, conformational dynamics different from that in the bulk solution was observed for the porous glass with $26 \mathrm{~nm}$ pore size. The characteristic effect of the confined geometry is larger in nonpolar cyclohexane than in polar DMF.

\section{References}

1. J. M. Drake and J. Klafter, Phys. Today, 46 (1990).

2. J. Klafter, A. Blumen and J. M. Drake, Molecular Dynamics in Restricted Geometries, eds. J. Klafter and J. M. Drake (John Wiley \& Sons, New York, 1989) pp. 1-22.

3. U. Even, K. Rademann, J. Jortner, N. Manor and R. Reisfeld, J. Luminescence, 31/32, 634 (1984).

4. P. Levitz, J. M. Drake and J. Klafter, J. Chem. Phys., 89, 5224 (1988).

5. D. Pines, D. Huppert and D. Avnir, J. Chem. Phys., 89, 1177 (1988); D. Pines and D. Huppert, Chem. Phys. Lett., 156, 223 (1989).

6. T. Bernstein, L. Kitaev, D. Michel and H. Pfeifer, J. Chem. Soc., Faraday Trans., I, 78, 761 (1982).

7. T. Fujii, A. Ishii, S. Suzuki and M. Anpo, Chemistry Exp., 4, 471 (1989).

8. L. L. Hench and J. K. West, Chem. Rev., 90, 33 (1990).

9. Dynamics in Small Confining Systems, eds. J. M. Drake, J. Klafter and R. Kopelman (Extended Abstracts, Materials Research Society, Boston, 1990).

10. W. D. D. Dozier, J. M. Drake and J. Klafter, Phys. Rev. Lett., 56, 197 (1986).

11. Shu Xu, J. Zhang and J. Jonas, J. Chem. Phys., 97, 4564 (1992).

12. J.-P. Korb, Shu Xu and J. Jonas, J. Chem. Phys., 98, 2411 (1993).

13. M. -C. Bellissent-Funel, J. Lal and L. Bosio, J. Chem. Phys., 98, 4246 (1993).

14. C. W. N. Cumper and A. P. Thurston, J. Chem. Soc. Perkin Trans., II, 106 (1972).

15. H. Miyasaka and N. Mataga, Ultrafast Phenomena VI, eds. C. B. Harris, E. P. Ippen, G. A. Mourou, A. H. Zewail (Springer-Verlag, Berlin, 1990) pp. 501-503.

16. R. W. Kessler and F. Wilkinson, J. Chem. Soc., Faraday Trans., 1, 77, 309 (1981).

17. M. Koshioka, H. Mizuma, K. Imagi, N. Ikeda, H. Fukumura, H. Masuhara and C. Krischi, Bull. Chem. Soc. Jpn., 63, 3495 (1990); N. Ikeda, K. Imagi, H. Masuhara, N. Nakashima and K. Yoshihara, Chem. Phys. Lett., 140, 281 (1987).

18. S. Hashimoto, N. Fukazawa, H. Fukumura and H. Masuhara, Chem. Phys. Lett., 223, 493 (1994).

19. A. Furube, Y. Matsuo, T. Asahi ad H. Masuhara, Abstracts of Ann. Rep. Chem. Soc. Jpn., 69, 1F239 (1995).

20. N. Tamai, T. Asahi and H. Masuhara, Rev. Sci. Instrum., 64, 2496 (1993).

21. N. Ikeda, M. Koshioka and H. Masuhara, Chem. Phys. Lett., 150, 452 (1988).

22. S. B. Dierker, B. S. Dennis and P. Wiltzius, J. Chem. Phys., 92, 1320 (1990).

23. M. A. Bell, B. Crystall, G. Rumbles, G. Porter and D. R. Klug, Chem. Phys. Lett., 222, 15 (1994). 